\title{
Deep Learning Approach for Sustainable WWTP Operation: A Case study on Data-Driven Influent 2 Conditions Monitoring
}

\author{
Abdelkader Dairi ${ }^{\mathrm{a}}$, Tuoyuan Cheng ${ }^{\mathrm{b}}$, Fouzi Harrou $^{\mathrm{c}}$, Ying Sun ${ }^{\mathrm{c}}$, TorOve Leiknes ${ }^{\mathrm{b}} \quad{ }_{4}$ \\ ${ }^{a}$ Computer Science Department, University of Oran 1 Ahmed Ben Bella, Algeria Street El senia 5 \\ el mnouer bp 31000 Oran \\ ${ }^{b}$ Water Desalination and Reuse Center (WDRC), Biological and Environmental Science and \\ Engineering (BESE) Division, King Abdullah University of Science and Technology (KAUST), \\ Thuwal, 23955-6900, Saudi Arabia, e-mail: tuoyuan.cheng@kaust.edu.sa \\ ${ }^{c}$ Computer, Electricaland Mathematical Sciences and Engineering (CEMSE) Division, King \\ Abdullah University of Science and Technology (KAUST), Thuwal, 23955-6900, Saudi Arabia, \\ e-mail: fouzi.harrou@kaust.edu.sa
}

Abstract

Wastewater treatment plants (WWTPs) are sustainable solutions to water scarcity. As initial conditions offered to WWTPs, influent conditions (ICs) affect treatment units states, ongoing processes mechanisms, and product qualities. Anomalies in ICs, often raised by abnormal events, need to be monitored and detected promptly to improve system resilience and provide smart environments. This paper proposed and verified data-driven anomaly detection approaches based on deep learning methods and clustering algorithms. Combining both the ability to capture temporal auto-correlation features among multivariate time series from recurrent neural networks (RNNs), and the function to delineate complex distributions from restricted Boltzmann machines (RBM), RNN-RBM models were employed and connected with various classifiers for anomaly detection. The effectiveness of RNN based, RBM based, RNN-RBM based, or standalone individual detectors, including expectation maximization clustering, K-means clustering, mean-shift clustering, one-class support vector machine (OCSVM), spectral clustering, and agglomerative clustering algorithms were evaluated by importing seven years ICs data from a coastal municipal WWTP where more than 150 abnormal events occurred. Results demonstrated that RNN-RBM-based OCSVM approach outperformed all other scenarios with an area under the curve value up to 0.98 , which validated the superiority in feature extraction by RNN-RBM, and the robustness in 
multivariate nonlinear kernels by OCSVM. The model was flexible for not requiring assumptions on data distribution, and could be shared and transferred among environmental data scientists.

Keywords: Wastewater treatment plant, Influent conditions monitoring, Machine 36 learning, Unsupervised deep learning

\section{Introduction}

For decades, the Kingdom of Saudi Arabia (KSA) has experienced a significant rise in both urbanization and population levels (Khan et al., 2017). The high living standards in KSA, a leading country from the Middle East, have exerted considerable pressure on energy service, water supply, and wastewater treatment. The annual gap between water supply and demand in KSA, which was around 11.5 billion $\mathrm{m}^{3}$, may not be eliminated by only enhancing energy-intensive desalination processes (da Silva et al., 2018; Lefers et al., 2016; Torregrossa et al., 2019). Therefore, wastewater treatment plants (WWTPs) that contributed to water resource reclamation have been given vital importance and are regarded as promising sustainable solutions to the water scarcity in KSA.

Resilient WWTPs have to sustain long-term qualified performance, despite temporally volatile volumes or qualities of the incoming wastewater (Haimi et al., 2013; Zhu and Anderson, 2017). Stringent discharge limits and rising operational costs, as well as intrinsic process nonstationarity and nonlinearity, posed challenges to operators (Corominas et al., 2017; Sweeney and Kabouris, 2014). As initial conditions offered to WWTPs, influent conditions (ICs) affect treatment units states, ongoing processes mechanisms, and product qualities. ICs accordingly were primary in operation thus emphasized, recorded, and monitored in KSA (Cheng et al., 2019). Anomalies or faults in ICs, often raised by abnormal events, need to be detected and diagnosed promptly to avoid facility failure, maintain effluent quality, support downstream operations, improve system resilience, reduce labor costs, and provide smart environments ( $\mathrm{Li}$ et al., 2017; Lorimer et al., 2018).

All over the years, methods were developed for prediction as well as fault de- $\quad 62$ tection and diagnosis, including mechanistic model-based (or analytical methods), $\quad 63$ and model-free (or data-driven methods) (Venkatasubramanian et al., 2003). An- 64 alytical models, based on ideal hypotheses, utilizing first principles, could theo- 65 retically explain system behavior, reveal process mechanism, but request prior hy- $\quad 66$ perparameters for calibration and would be questioned by costly high-dimensional $\quad 67$ 
computation and ill-conditioned problems (Haimi et al., 2013). Data-driven methods may perform systematic and objective exploration, visualization, and interpretation of data, identify essential factors, features or patterns, and endorse then optimize data-supported decision-making (Gibert et al., 2018a). Validated datadriven monitoring methods could be transferred and shared conveniently among domain experts, due to the versatile nature of data science models (Gibert et al., 2018b). Though we have stronger computation power nowadays, due to the limited investments in instrumentation, control, and automation in WWTPs, and the lack of data science background for WWTP professionals, it could be demanding to make the best of the data (Newhart et al., 2019; Yuan et al., 2019). Today, datadriven methods have been more common in the environmental field, even though not as widespread as, for example, in petrochemical industry where soft-sensors have been extensively utilized since billions of dollars were once wasted annually due to abnormal events (Venkatasubramanian et al., 2003). Environmental data have been adopted by data-driven methods for anomaly detection in meteorological signals (Hill and Minsker, 2010), prediction of downstream pollutants concentration in river networks (Álvarez-Cabria et al., 2016), and sludge bulking monitoring in WWTPs (Capodaglio et al., 1991).

Traditionally in data-driven modeling, time series analyses have widely been developed to model WWTPs (Kazor et al., 2016). Boyd et al. (2019) validated autoregressive integrated moving average (ARIMA) models in daily influent flow forecasting for five stations across North America. Li et al. (2019) improved autoregressive with exogenous inputs models to predict inflows at sewage pumping stations with different hydraulic characteristics and climatic conditions. Man et al. (2019) proposed an approach to forecast COD load in municipal sewage based on ARIMA and vector AR algorithms. Xiao et al. (2017) applied multistep ARMA model to monitor and diagnose ICs.

Machine learning is a remarkable multidisciplinary field, where methods could be implemented for fault detection. A traditional artificial neural network (ANN) simulation for the monitoring and control of an anaerobic WWTP was reported (Wilcox et al., 1995). Neural fuzzy models together with ANN were applied for monitoring and predicting WWTPs (Dias et al., 2007). ANN models have also been researched to monitor multiple key parameters including oxidation-reduction potential and UV light intensity in WWTPs (Lin et al., 2012). K-nearest neighbor methods were tested to predict influent conditions (Kim et al., 2016). Hybrid approaches combining ANN with multiple linear regression were researched to forecast biochemical oxygen demand of the influent, which was expensive and 
difficult to measure with traditional hard sensors (Zhu et al., 2018).

Deep learning methods have gained much attention from researchers recently for their power to extract knowledge from large and complex datasets (Deutsch and He, 2018; Oneto et al., 2017; Wang et al., 2016; Yang et al., 2018). In the literature, numerous deep learning architectures have been employed to address practical problems. Among them, one consensus was that recurrent neural networks (RNNs) can capture long-term dependencies (Boulanger-Lewandowski et al., 2012; Martens and Sutskever, 2011). Their outputs were based on the actual and past sequential information of inputs (Czuszynski et al., 2018). Therefore, algorithms based on RNNs were proven to be effective in numerous applications including motion tracking of robot manipulators (Xiao et al., 2018), polyphonic music generation and transcription (Boulanger-Lewandowski et al., 2012), predicting stock market trends (Yoshihara et al., 2014), intrusion detection(Sheikhan et al., 2012), and gesture recognition (Czuszynski et al., 2018). RNN-based approaches have also been employed to improve sustainable WWTP operations. For instance, Qiao et al. (2012) designed an RNN-based approach to control the dissolved oxygen concentration, nitrate-nitrogen concentration and mixed liquor suspended solids concentration in a WWTP. Baruch et al. (2005) applied RNN in modeling and adaptive control of WWTP processes.

Recently, an improved extension of RNN has been developed by integrating RNN with the restricted Boltzmann machine (RBM). The RNN-RBM permitted freedom to delineate the temporal correlations involved and merged desirable properties of both RNNs and RBMs to handle dependencies in time series (Boulanger-Lewandowski et al., 2012). Specifically, the RNN-RBM model would employ the capability of the RBMs to describe complicated distributions and the efficiency of RNNs to model long-term dependencies. The RNN-RBM model was initially introduced by Boulanger-Lewandowski et al. (2012) to discover temporal dependencies in high-dimensional sequences from polyphonic music generation and transcription. RNN-RBM has also successfully predicted the evolution of traffic congestion in large-scale transportation networks (Ma et al., 2015). Till now, RNN-RBM models, however, have not been employed to monitor ICs from WWTPs.

In this study, in order to monitor and detect abnormal influent conditions for sustainable and resilient wastewater treatment plant operations, we developed data-driven unsupervised anomaly detection approaches based on deep learning methods and clustering algorithms. The coupled RNN-RBM methodology may potentially carry both flexibility and feature extraction capacity, that could de- 
scribe temporal dependencies in multivariate time series, and therefore would im- 142 prove detection performance of following classification models such as agglom- ${ }_{143}$ erative clustering (AGC), expectation maximization (EM) clustering, k-means ${ }_{144}$ (KM), mean-shift (MS), one-class support vector machine (OCSVM), and spec- ${ }_{145}$ tral clustering (SC). Real ICs dataset collected from a coastal municipal WWTP ${ }_{146}$ located in Saudi Arabia is adopted to evaluate suggested algorithms. To the best ${ }_{147}$ of our knowledge, this is the first investigation case in anomaly detection using ${ }_{148}$ RNN-RBM with OCSVM on ICs of WWTPs.

The deep Boltzmann machines (DBM), RNN-RBM methods were introduced 150 in Section 2. The proposed monitoring schemes were described in Section 3. ${ }_{151}$ The performance of the recommended approaches were evaluated via real data in $\quad 152$ Section 4, and conclusions were drawn in Section 5.

\section{Preliminaries}

This section provided an overview of the DBM, recurrent temporal RBM and ${ }_{155}$ coupled RNN-RBM models.

\subsection{Deep Boltzmann machine (DBM)}

157

Deep Boltzmann machine was initially introduced as an extension of restricted 158 Boltzmann machine with multiple hidden layers (Salakhutdinov and Hinton, 2009). 159 DBM was categorized as Markov random field and generative model, since it 160 could be represented by an undirected graph in which deep layers of hidden vari- ${ }_{161}$ ables were undirectly connected (illustrated in Figure 1). Generally speaking, 162 with deepen architectures, DBM acquired the enhanced ability to discover and ${ }_{163}$ learn well complex statistical structures (representations) of the inputs.

The DBM was trained in an unsupervised manner with unlabeled data. Salakhut- 165 dinov and Hinton (2009) investigated a greedy, layer-by-layer pre-training ap- 166 proach, which began by learning a stack of RBMs using the modified Contrastive ${ }_{167}$ divergence algorithm. The DBM was constructed to offer a richer model than 168 an RBM for discovering relevant features in complex data by incorporating more ${ }_{169}$ layers of hidden units. Probability distributions could be defined by energy func- 170 tions in energy-based probabilistic models. The DBM energy function of the state 171 $\left\{v, h^{(1)}, h^{(2)}\right\}$ was:

$$
E\left(v, h^{(1)}, h^{(2)} ; \theta\right)=-v^{T} W^{(1)} h^{(1)}-h^{(1)} W^{(2)} h^{(2)}
$$




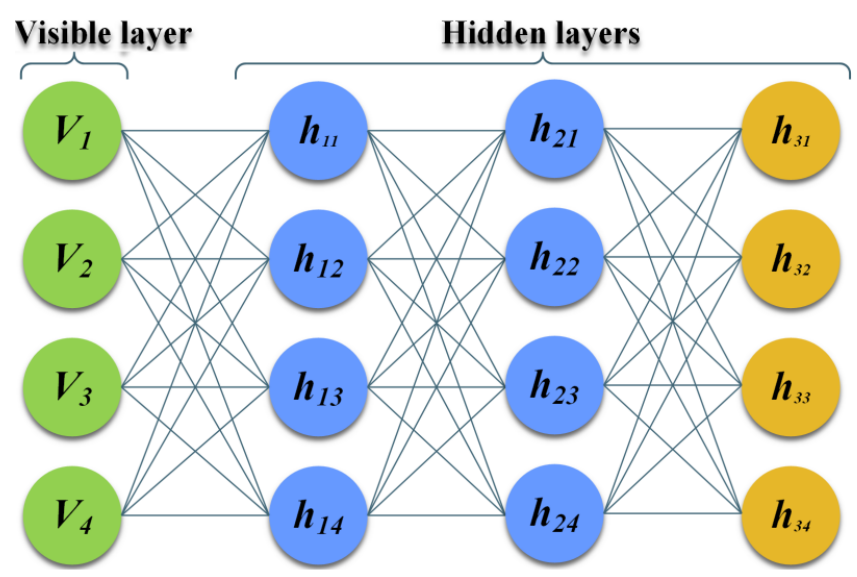

Figure 1: Diagram of a deep Boltzmann machine (DBM).

where $\theta$ defined the model parameters as $\theta=\left\{W^{(1)}, W^{(2)}\right\}$, the vector of visible $\quad 173$ units was $v \in\{0,1\}^{D}$ and the vectors of hidden units were $h^{(1)}, h^{(2)} \in\{0,1\}^{P}$. ${ }^{174}$ The join probability distribution assigned by the model to $v$ was:

$$
p(v ; \theta)=\frac{1}{Z(\theta)} \sum_{h^{(1)}, h^{(2)}} \exp \left(-E\left(v, h^{(1)}, h^{(2)} ; \theta\right)\right)
$$

The learning process of an DBM was achieved by minimization of the nega- $\quad 176$ tive log-likelihood, where its derivative with respect to the model parameters was 177 expressed in the following form:

$$
\frac{\partial \log P(v ; \theta)}{\partial W^{(1)}}=\mathbb{E}_{P_{\text {data }}}\left[v h^{(1) T}\right]-\mathbb{E}_{P_{\text {model }}}\left[v h^{(1) T}\right]
$$

where $\mathbb{E}_{P_{\text {model }}}[$.$] denoted an expectation with respect to the distribution defined 179$ by the model,and $\mathbb{E}_{P_{\text {data }}}[$.$] was an expectation with respect to the completed data 180$ distribution.

\subsection{Recurrent temporal RBM}

Traditional neural networks started processing the input assuming the absence ${ }_{183}$ of dependencies, neither between the observed data nor the outputs. However, in ${ }_{184}$ real practices, temporal dependence on historical data existed in time series data. $\quad 185$ In other words, the generated output from the network at time instant $t-1$ would ${ }_{186}$ impact the decision at time instant $t$. To cope with the limitations, recurrent neural ${ }_{187}$ networks were introduced (Williams and Zipser, 1989) and had become powerful ${ }_{188}$ 
tools for time-dependent modeling. RNNs permitted discovery and learning of ${ }_{189}$ temporal dependences by incorporating the previous output as inputs. This al- 190 gorithm was incorporating not only current inputs but also recent past inputs, to 191 produce new outputs (Figure 2).

The topology of RNN was based on nonlinear stacked units, where at con- 193 nections between units formed a directed cycle (Figure 2). RNN was deep in 194 time steps when unrolled (unfolded over time). This structure enhanced feature 195 discovering and learning of long-term dependencies from sequential time-series 196 data, which made RNNs appropriate for dynamic system modeling.

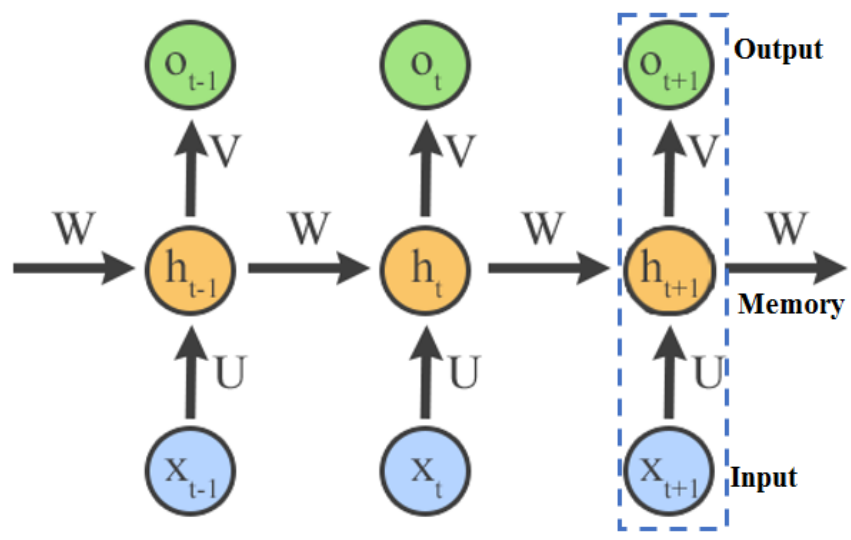

Figure 2: Diagram of a recurrent neural network (RNN).

The recurrent temporal RBM (RTRBM) (Sutskever et al., 2009) was a succes- 198 sion of conventional RBMs (Figure 3). The parameters of RTRBM models were 199 time-dependent, where their dependencies were on the sequential history at time 200 $t$. We denoted the RTRBM parameters respectively as $b_{v}, b_{h}, W^{(t)}$, and

$$
\mathcal{M}^{(t)} \equiv\left\{v^{(\tau)}, \hat{h}^{(\tau)} \mid \tau<t\right\}
$$

where $\hat{h}^{(t)}$ was the mean-field value of $h^{(t)}$, and the biases depended on $\hat{h}^{(t-1)}$. $\quad 202$ The joint probability distribution of RTRBM was defined by (Sutskever et al., 203 2009):

$$
P\left(v^{(t)}, h^{(t)}\right)=\prod_{t=1}^{T} P\left(v^{(t)}, h^{(t)} \mid \mathcal{M}^{(t)}\right)
$$


Single arrows represent a deterministic function, double arrows represent the stochastic hidden-visible connections of an RBM.

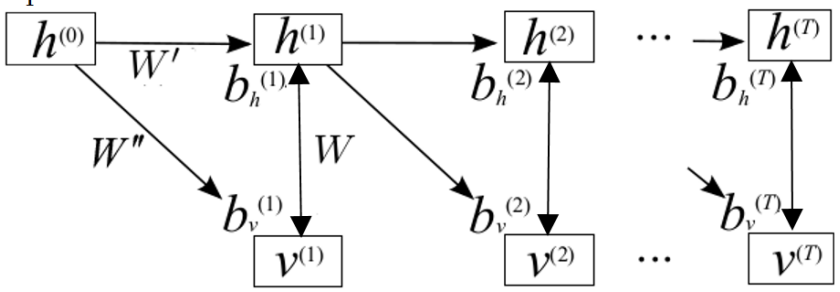

Figure 3: Diagram of a recurrent temporal RBM.

In Eq.5, $P\left(v^{(t)}, h^{(t)} \mid \mathcal{M}^{(t)}\right)$ was the joint probability of the $t^{t h}$-RBM, with Eq.6, 7205 defining its parameters.

$$
\begin{aligned}
& b_{h}=b_{h}+W^{\prime} \hat{h}^{(t-1)} \\
& b_{v}=b_{v}+W^{\prime \prime} \hat{h}^{(t-1)}
\end{aligned}
$$

In summary, RTRBM had six parameters: $W, b_{v}, b_{h}, W^{\prime}, W^{\prime \prime}, \hat{h}^{(0)}$. Note that 207 the Eq.8 defined a single-layer RNN, with hidden units $\hat{h}^{(0)}$

$$
\hat{h}^{(t)}=\sigma\left(W v^{(t)}+b_{h}\right)=\sigma\left(W v^{(t)}+W^{\prime} \hat{h}^{(t-1)}+b_{h}\right)
$$

\subsection{Coupled RNN-RBM approach}

Coupled RNN-RBM model, as an extension of the recurrent temporal RBM, was integrating both models in order to capture and predict temporal dependen- 211 cies in high-dimensional data (Boulanger-Lewandowski et al., 2012). Generally 212 speaking, as an energy-based model, the RNN-RBM augmented the RNN model 213 by including an RBM at every time step (Boulanger-Lewandowski et al., 2012). 214 The configuration was constructed based on an RBM, whose model parameters 215 were determined from an RNN (Figure 4).

The hidden unit $\hat{h}^{(t)}$ of the RNN layer at time instant $t$ was directly linked to ${ }_{217}$ its predecessor $\hat{h}^{(t-1)}$ and to $V^{t}$ as shown in the Eq. 9:

$$
\hat{h}^{(t)}=\sigma\left(W_{2} v^{(t)}+W_{3} \hat{h}^{(t-1)}+b_{\hat{h}}\right)
$$

where the parameters of a single-layer RNN-RBM were: $W, b_{v}, b_{h}, W^{\prime}, W^{\prime \prime}, \hat{h}^{(0)}, \quad{ }^{219}$ $W_{2}, W_{3}$ and $b_{\hat{h}}$. The joint probability distribution of RNN-RBM was obtained 220 


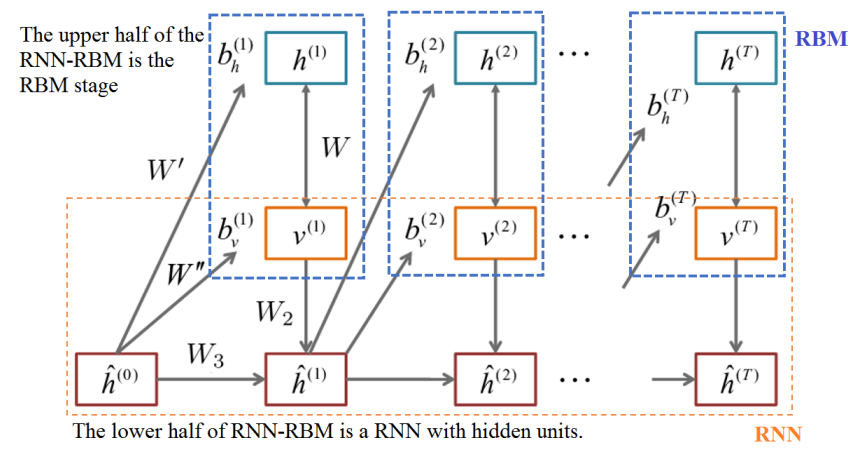

Figure 4: Diagram of a single-layer RNN-RBM.

by placing $\hat{h}^{(t)}$ in Equation (5). In fact, RTRBM was constrained by the hidden units needed to represent the conditional distributions and to transmit temporal information. Combining a complete RNN with distinct hidden units $\hat{h}^{(t)}$ would overcome this constraint.

\section{RNN-RBM-based OCSVM anomaly detection approach}

225

In this section, the outline and the training procedure of the RNN-RBM model 226 were presented. The principles of connecting OCSVM with RNN-RBM models ${ }_{227}$ to efficiently detect anomalies in multivariate time-series data were illustrated. $\quad{ }_{228}$

Aiming at describing dynamics and autocorrelation in multivariate time series 229 such as ICs data of WWTP, we adopted the data-driven RNN-RBM modeling 230 approach. This method could employ RNN-conditioned RBM as the powerful ${ }_{231}$ data distribution estimator to depict local and long-term dependences contained ${ }_{232}$ in the dataset (Boulanger-Lewandowski et al., 2012).

In the training step of the RNN-RBM model, anomaly-free data were inputted ${ }_{234}$ in an unsupervised manner, to obtain a feature space that may reconstruct the input ${ }_{235}$ with limited error via encoding and decoding tasks. The designed and trained 236 RNN-RBM model could thereafter be applied to predict the evolution of inflow ${ }^{237}$ conditions of WWTPs. During the training phase, cross-entropy error was set ${ }_{238}$ as the cost function to minimize (Vincent et al., 2010). This cost function could 239 quantify the accuracy of the model when representing inputs in the training set, 240 by calculating the dissimilarity between the probability distributions of input and 241 reconstruction from the RNN-RBM model. The cross-entropy was given as: 242 


$$
\mathcal{C}(X, \hat{X})=-\sum_{i}^{n}\left(\hat{X}_{i} \log \left(X_{i}\right)+\left(1-\hat{X}_{i}\right) \log \left(1-X_{i}\right)\right)
$$

where $X$ was the distribution of input data and $\hat{X}$ was the distribution of recon- ${ }^{243}$ structed data from the fitted model. The initialization of parameters played a vital 244 role in reaching the global minimum of the cost function. Here, the initial values 245 of deep learning parameters have been tuned in manual manner in the training 246 stage.

Then the designed RNN-RBM model together with clustering algorithms (i.e., 248 EM, KM, MS, OCSVM, SC, and AGC) were adopted to detect abnormalities in 249 multivariate IC time series data (see Figure 5). Here, the output features from 250 the RNN-RBM model were used by the classifiers to differentiate or discriminate 251 between normal and abnormal patterns.

In summary, the proposed RNN-RBM-based anomaly detection approachwas 253 consisted of three stages: 1) Unsupervised RNN-RBM model construction using 254 time-series training dataset composed of normal observation (with no anomaly). 255 2) Unsupervised classifiers (i.e., EM, KM, MS, OCSVM, SC, and AGC) training 256 using features discovered by the RNN-RBM model. 3) Anomaly detection on 257 testing dataset using the constructed models. Key steps of the proposed procedure 258 are outlined in Table 1.

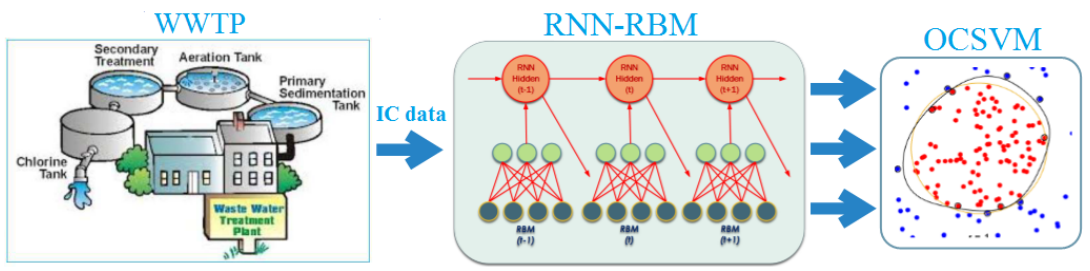

Figure 5: RNN-RBM-based OCSVM approach for anomaly detection.

\section{Results and discussion}

To test the proposed schemes for IC monitoring with real dataset, historical 262 ICs from the WWTP based in King Abdullah University of Science and Technol- ${ }_{263}$ ogy (KAUST), Thuwal, Saudi Arabia (Figure 6) were engaged. This plant had a 264 sustainability mission to provide all treated effluents for irrigation reuse among 265 
Table 1: Main steps in building recurrent probabilistic feature extractors of the proposed system.

Phase 1: Offline modeling

Step 1 Data (Time series) acquisition and normalization. Abnormal events were reported from operators and labeled as anomalies.

Step 2 Discovering learning model parameters time dependency via unsupervised training of the RNN-RBM, where only one RBM was used at each time-step.

Step 3 Computing the corresponding initialization of different weights and bias for RNN-RBM.

Step 4 Generating new data representation through learned features from the trained RNN-RBM model.

Step 5 Training classifiers with the dataset obtained in the previous step.

Step 6 Defining the hyperplane that separated inliers from outliers, which functioned as the anomaly detector.

Phase 2: Anomaly detection

Step 1 Collection of new observation $\mathcal{X}$.

Step 2 Normalization of the $\mathcal{X}$ to $\hat{\mathcal{X}}$.

Step 3 Mapping $\hat{\mathcal{X}}$ into feature space $\mathcal{F}_{\hat{\mathcal{X}}}$ using trained RNN-RBM model.

Step 4 Classification of $\mathcal{F}_{\hat{\mathcal{X}}}$ as inlier (normal) or outlier (abnormal) based on each hyperplanes or detectors.

the campus, which greatly reduced potable water demand of the university. How- 266 ever, more than 150 real abnormal events occurred, such as intensive rainfalls, 267 seawater intrusion into the lift station, discharge from construction area over the 268 limit, and hypochlorite dosage (see Table 2). All of them caused effects on the 269 WWTP operation (downstream processes compared to the inflow) therefore are 270 identified and reported by operators. Abnormal events reported from operators, 271 as marked in their daily log, when they were examining historical data after the 272 event, were recognized and labeled as anomalies, by which binary classification 273 scenarios (anomaly detection) were formed. By involving data-driven machine 274 learning based monitoring techniques, we hoped to recognize faults in the influ- 275 ent, make decisions and take action before they flow into the process, and upgrade 276 sustainability of the plant.

Operators maintained daily sampling of the inflow from headwork, on which 278 physical, chemical analysis, and recording are performed. The recorded dataframe 279 contains seven years (from Sep. 1st, 2010 to Sep. 1st, 2017) of daily measure- 280 
ments over twenty-one variables (Table 3) with few not available entry (within 1\%, 281 132 out of 63950). Variables were measured by at-line sensors, and recorded daily 282 by operators. Sensors produced by YSI, Xylem Analytics Co. were maintained 283 and calibrated daily to achieve reliable performance. The Pearson's correlation co- ${ }^{284}$ efficients from the standard curve of measurements from sensors versus ones from 285 traditional methods were inspected bimonthly to be over 0.90 , as reported from 286 the local technician. This study proposed a monitoring method for influent con- ${ }^{287}$ ditions, which was at the very beginning of the whole process and may give early ${ }_{288}$ warning to operators and facilitate control in downstream processes. Typically, 289 the hydraulic retention time (or reaction time in the whole facility, from headwork 290 to discharge) in a WWTP was long, in comparison with the time required to mea- 291 sure, compute, raise alarm, and take action, which validated the monitoring of 292 influent and its potential for proper control in this industry. To cope with the miss- 293 ingness, and to avoid dropping all partially observed events from the analysis that 294 would result in biases, inefficiencies, and uncertainty, we adopted the R package 295 Amelia for imputation, in which multiple imputations were performed (Honaker 296 et al., 2011). It has been shown that, as an alternative to listwise deletion, multiple imputations could reduce bias and increase efficiency. The algorithms employed both bootstrapping and expectation maximization, whose details are delineated by Honaker and King (2010). In this case study, 100 groups of computations were carried in parallel, and averaged to yield final values.

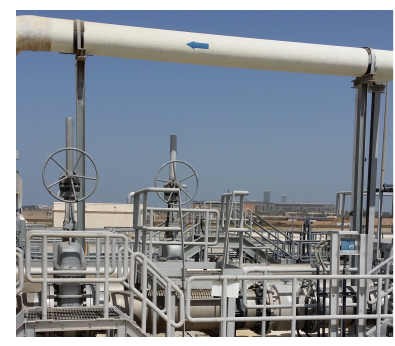

(a)

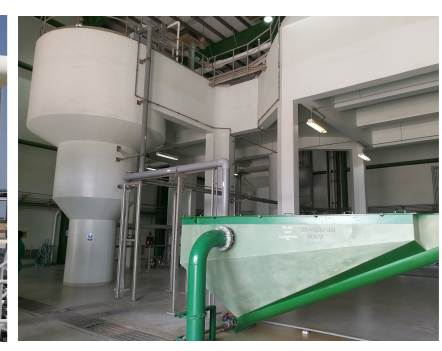

(b)

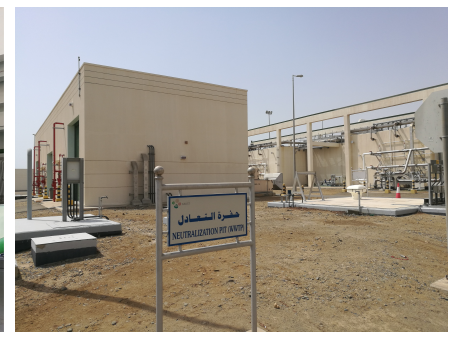

(c)

Figure 6: a) The pipeline from the campus, b) the grit chamber and grit classifier inside the headwork, c) the neutralization pit.

The training dataset from September 1st, 2010 to May 14, 2011, were used 302 to construct the RNN-RBM model. Instead of displaying all observations of a 303 dataset or only mean with standard deviation, the Table 4 presented summary val- 304 ues of the training dataset: the minimum, the first quartile, the median, the third 305 
Table 2: Anomalies in the testing dataframe.

\begin{tabular}{lr}
\hline Fault types & Counts \\
\hline Discharge from construction area & 3 \\
InFlow-Total (Rainfall) & 5 \\
Internal circulation & 3 \\
Lift station maintenance/flushing & 2 \\
Lime dumping from RO plant & 9 \\
NaOCl dumping from RO plant & 26 \\
Others & 6 \\
pH over limit & 9 \\
Seawater Intrusion & 45 \\
TDS over limit & 11 \\
Total alkalinity over limit & 36 \\
Water supply shutdown & 2 \\
\hline Total & 157 \\
\hline
\end{tabular}

Table 3: Monitored influent conditions.

\begin{tabular}{lll}
\hline No. & Variable name & Measurement scopes \\
\hline 1 & InFlow-LS1 & Wastewater inflow, from the whole campus area, in $\mathrm{m}^{3} /$ day \\
2 & InFlow-LS8 & Wastewater inflow, from a desalination plant, in $\mathrm{m}^{3} /$ day \\
3 & InFlow-DP & Wastewater inflow, recycled from WTTP itself, in $\mathrm{m}^{3} /$ day \\
4 & InFlow-Total & Wastewater inflow, from the whole university, in $\mathrm{m}^{3} /$ day \\
5 & Temp & Temperature, in Celsius \\
6 & $\mathrm{pH}$ & Potential of hydrogen, unitless \\
7 & $\mathrm{Conductivity}$ & Conductivity, in $\mu \mathrm{S} / \mathrm{cm}$ \\
8 & $\mathrm{TDS}$ & Total dissolved solid, in $\mathrm{mg} / \mathrm{L}$ \\
9 & $\mathrm{TSS}$ & Total suspended solid, in $\mathrm{mg} / \mathrm{L}$ \\
10 & $\mathrm{CaHardness}$ & Calcium hardness, in $\mathrm{mg} / \mathrm{L}$ \\
11 & $\mathrm{MgHardness}$ & Magnesium hardness, in $\mathrm{mg} / \mathrm{L}$ \\
12 & TotalAlkalinity & Total alkalinity, in $\mathrm{mg} / \mathrm{L}$ \\
13 & $\mathrm{BOD}_{5}$ & 5-day biochemical oxygen demand, in $\mathrm{mg} / \mathrm{L}$ \\
14 & $\mathrm{COD}$ & Chemical oxygen demand, in $\mathrm{mg} / \mathrm{L}$ \\
15 & $\mathrm{FOG}_{16}$ & Fat, oils and grease, in $\mathrm{mg} / \mathrm{L}$ \\
17 & $\mathrm{TKN}_{3} \mathrm{~N}$ & Total Kjeldahl nitrogen, in $\mathrm{mg} / \mathrm{L}$ \\
18 & $\mathrm{NO}_{3} \mathrm{~N}$ & Ammonia, in $\mathrm{mg} / \mathrm{L}$ \\
19 & $\mathrm{PO}_{4} \mathrm{P}$ & Nitrate, in $\mathrm{mg} / \mathrm{L}$ \\
20 & $\mathrm{Cl}_{21}$ & Phosphate, in $\mathrm{mg} / \mathrm{L}$ \\
21 & $\mathrm{Boron}$ & Chloride, in $\mathrm{mg} / \mathrm{L}$ \\
\hline & & Boron, in $\mathrm{mg} / \mathrm{L}$ \\
\hline
\end{tabular}


quartile, the maximum, kurtosis, and skewness. Skewness and kurtosis were cal- 306 culated to show the symmetry and shape of the studied time series distributions. 307 From Table 4, we may conclude that several IC variables were positively skewed 308 with strong kurtosis. The training set was normalized after smoothing with the ex- 309 ponentially weighted moving average method to reduce noise effects. Parameters 310 of the constructed RNN-RBM and OCSVM were presented in Table 5.

311

Table 4: Descriptive statistics of the training dataframe.

\begin{tabular}{llllllllll}
\hline & mean & std & $\min$ & 0.25 & 0.5 & 0.75 & max & skewness & kurtosis \\
\hline InFlow-LS1 & 3021.61 & 535.45 & 2228.00 & 2660.00 & 2851.00 & 3244.00 & 5249.00 & 1.33 & 1.89 \\
InFlow-LS8 & 279.31 & 156.08 & 64.00 & 156.00 & 228.00 & 366.00 & 867.00 & 1.13 & 0.92 \\
InFlow-DP & 47.43 & 95.79 & 0.00 & 9.00 & 10.00 & 36.00 & 749.00 & 3.80 & 18.67 \\
InFlow-Total & 3512.92 & 611.00 & 2558.00 & 3036.00 & 3389.00 & 3853.00 & 5642.00 & 0.86 & 0.34 \\
Temp & 29.31 & 1.59 & 25.99 & 28.10 & 29.20 & 30.60 & 32.50 & 0.27 & -0.85 \\
pH & 7.40 & 0.25 & 6.59 & 7.25 & 7.36 & 7.52 & 8.56 & 0.91 & 3.20 \\
Conductivity & 669.37 & 284.69 & 264.00 & 537.00 & 625.00 & 719.00 & 2466.00 & 3.19 & 15.37 \\
TDS & 459.48 & 209.08 & 174.00 & 363.00 & 429.00 & 492.00 & 1809.00 & 3.37 & 16.69 \\
TSS & 68.66 & 27.29 & 12.00 & 49.00 & 64.00 & 82.00 & 187.00 & 1.14 & 2.10 \\
CaHardness & 72.75 & 30.78 & 20.00 & 52.00 & 72.00 & 94.00 & 176.00 & 0.49 & 0.17 \\
MgHardness & 41.91 & 27.46 & 6.00 & 24.00 & 36.00 & 48.00 & 156.00 & 1.81 & 3.59 \\
TotalAlkalinity & 120.88 & 24.98 & 68.00 & 100.00 & 120.00 & 136.00 & 196.00 & 0.22 & -0.33 \\
BOD & 99.03 & 36.95 & 27.00 & 71.00 & 92.00 & 123.00 & 224.00 & 0.58 & 0.10 \\
COD & 152.99 & 61.83 & 42.00 & 102.00 & 157.00 & 189.00 & 329.00 & 0.50 & -0.25 \\
FOG & 54.36 & 53.05 & 2.90 & 14.30 & 37.10 & 77.10 & 351.40 & 1.86 & 5.25 \\
$\mathrm{TKN}$ & 17.91 & 6.18 & 2.10 & 13.80 & 17.30 & 21.90 & 37.90 & 0.30 & 0.45 \\
$\mathrm{NH}_{3} \mathrm{~N}$ & 11.84 & 4.10 & 0.94 & 9.30 & 12.00 & 14.50 & 23.60 & -0.06 & 0.47 \\
$\mathrm{NO}_{3} \mathrm{~N}$ & 4.17 & 1.68 & 0.10 & 2.90 & 4.20 & 5.10 & 9.80 & 0.49 & 0.47 \\
$\mathrm{PO}_{4} \mathrm{P}$ & 8.25 & 2.86 & 1.30 & 6.40 & 8.10 & 10.00 & 23.50 & 1.41 & 6.59 \\
$\mathrm{Cl}$ & 126.09 & 75.62 & 45.00 & 91.00 & 107.00 & 137.00 & 654.00 & 4.29 & 23.37 \\
$\mathrm{Boron}$ & 1.15 & 0.33 & 0.50 & 0.90 & 1.10 & 1.30 & 2.50 & 1.40 & 2.63 \\
\hline
\end{tabular}

312 The cross-entropy measure was involved in analyzing the accuracy of the con- 312 313 structed model. The stopping rule of training phase would be reached when re- 313 314 constructions were adequately approximating original inputs. Calculated cross- 314 315 entropy values along epochs during the training stage displayed that the recon- 315 316 structed error shrank rapidly with increasing iterations, and converged success- 316 317 fully within 50 steps, validating sufficient learning under the current configura- ${ }_{317}$ 318 tions, for each hyperparameter set. 318

319 The testing dataset, with faults, covered the period from May 15, 2011, to 319 320 September 1st, 2017. Detection performance of developed approaches was cal- 
Table 5: Parameters in RNN-RBM and OCSVM.

\begin{tabular}{l|ll}
\hline Models & Parameter & Value \\
\hline RNN-RBM & batch size & 10 \\
& learning rate & 0.001 \\
& loss function & cross-entropy \\
& number of hidden units by layer & 40 \\
& number of recurrent hidden units & 20 \\
& number of visible units by layer & 20 \\
& optimizer & Adam \\
& training epochs & 200 \\
\hline OCSVM & gamma & 2 \\
& kernel & radial basis function \\
& nu & 0.001 \\
\hline
\end{tabular}

culated upon the testing dataset. Aside from the OCSVM above, comparisons 321 from the RNN-RBN-based expectation maximization clustering (Dempster et al., 322 1977), K-means clustering (Arthur and Vassilvitskii, 2007), mean-shift clustering, spectral clustering, and agglomerative clustering algorithms were also computed 324 and displayed. In the expectation maximization clustering algorithm, the fitting 325 between given data and Gaussian mixture models was optimized (Dempster et al., 326 1977). In such cases, binary clustering algorithms were applied to the features 327 extracted by the RNN-RBN model for anomaly detection. They were trained with 328 only anomaly-free data, but without involving their labels in learning. Most of the ${ }_{329}$ clustering algorithms here were parametric, on which two clusters were imposed 330 in the training period, during which normal observations would regroup into a 331 dominant cluster automatically since they share similar features. Besides, for the 332 clustering methods that we can not specify the number of clusters, the same rule ${ }_{3}$ was applied by considering only the dominant cluster since it regroups the major ${ }_{334}$ elements of the observations. A self-evident assumption is that, unsupervised al- ${ }_{335}$ gorithms would capture the most representative dominant features of the majority 336 observations, which should be the 'most' normal conditions. In other words, we ${ }_{337}$ are assuming normal conditions consisted most of the observations, and would 338 exhibit prevalence in clustering, without prior labeling judgment. Thereafter, in $3 з 9$ the testing phase, easily via existing clusters they could cluster and classify the 340 test data as inlier (normal observation) or outlier (anomaly). 341

Parameters of the studied algorithms were presented in Table 6. Here, the val- 342 ues of the clustering algorithm parameters are determined via grid search in the 343 training phase. Moreover, performances of detection procedures were quantita- ${ }_{344}$ 
sion and recall), and area under the curve (AUC).
345 rate (FPR or recall), accuracy, precision, F1 score (the weighted average of preci- 346

347

Table 6: Parameters in other clustering algorithms.

\begin{tabular}{|c|c|c|}
\hline Models & Parameter & Value \\
\hline RBM & $\begin{array}{l}\text { Learning rate } \\
\text { Training epochs } \\
\text { batch size } \\
\text { loss function } \\
\text { number of hidden units by layer } \\
\text { number of visible units by layer } \\
\text { optimizer }\end{array}$ & $\begin{array}{l}0.001 \\
200 \\
10 \\
\text { cross-entropy } \\
40 \\
20 \\
\text { SGD }\end{array}$ \\
\hline DBM & $\begin{array}{l}\text { Layers } \\
\text { Learning rate } \\
\text { Training epochs } \\
\text { loss function } \\
\text { number of hidden units by layer } \\
\text { number of visible units by layer } \\
\text { optimizer }\end{array}$ & $\begin{array}{l}03 \\
0.001 \\
200 \\
\text { cross-entropy } \\
40 \\
20 \\
\text { SGD }\end{array}$ \\
\hline $\mathrm{AGC}$ & $\begin{array}{l}\text { Affinity } \\
\text { Linkage }\end{array}$ & $\begin{array}{l}\text { Euclidean } \\
\text { Ward }\end{array}$ \\
\hline EM & $\begin{array}{l}\text { Covar } \\
\text { Covariance type } \\
\text { Iteration }\end{array}$ & $\begin{array}{l}1 \mathrm{e}-06 \\
\text { Full } \\
100\end{array}$ \\
\hline KMeans & $\begin{array}{l}\text { Init } \\
\text { Iteration }\end{array}$ & $\begin{array}{l}10 \\
300\end{array}$ \\
\hline Mean-Shift & Bandwidth & 0.44 \\
\hline Spectral Clustering & $\begin{array}{l}\text { Affinity } \\
\text { Gamma }\end{array}$ & $\begin{array}{l}\text { RBF } \\
1.0\end{array}$ \\
\hline
\end{tabular}

tively evaluated using following metrics: true positive rate (TPR), false positive

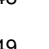

\section{det}

Ris

\section{Ods} the

\section{of} spaces.

Monitoring results of the studied approaches were displayed in Figure 7, while 348 detection performances were summarized in Table 7. As clearly shown, the detec- 349 tion efficiency and accuracy were greatly enhanced by using the proposed RNN- 350 RBM-based OCSVM approach. Indeed, OCSVM outperformed the other meth- 351 ods (Table 7) by achieving an AUC up to 0.98, which flagged its ability to detect 352 he vast majority of abnormal events reported by the operator, while avoiding 353 raising false alarms at the same time. This fact was attributed to the flexibility 354 f RNN-RBM models and the sensitivity of OCSVM algorithms, that could iden- 355 fy deviation from normal situations both sensitively and accurately in the feature 356 357 
(a)

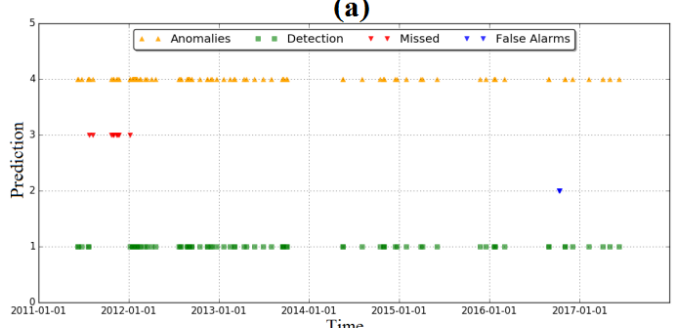

(c)

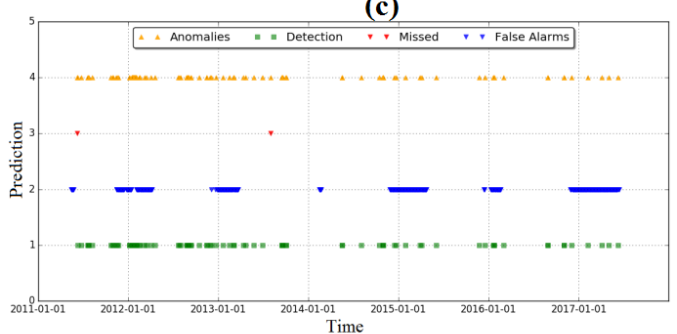

(e)

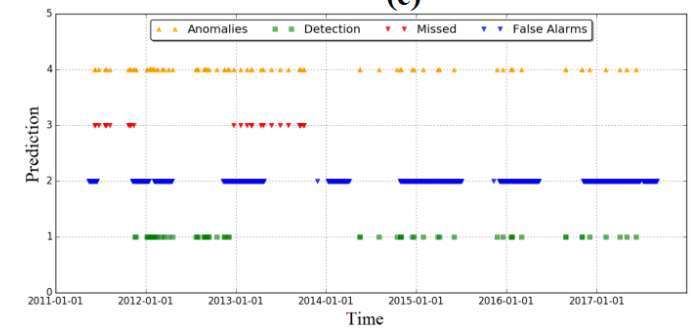

(b)

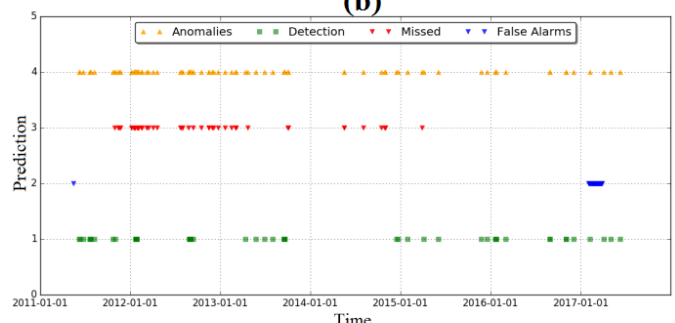

(d)

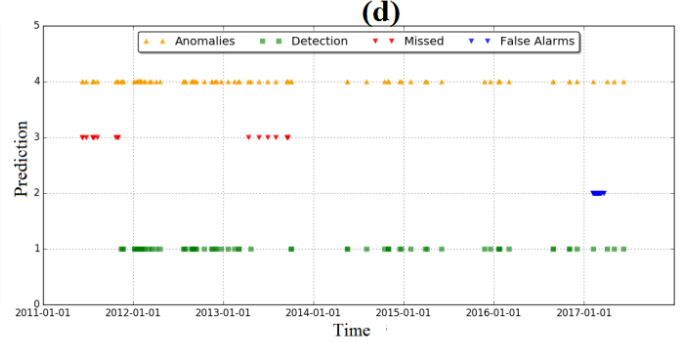

Figure 7: Monitoring results of RNN-RBM-based (a) one-class support vector machine, (b) expectation maximization clustering, (c) K-means clustering, (d) spectral clustering, and (e) agglomerative clustering.

Other deep probabilistic methods, such as RBM, DBM and RNN were im- 358 plemented individually for comparison. In these RBM, DBM and RNN -based 359 approaches, binary clustering schemes (i.e., AGC, EM, KM, MS, OCSVM, SC) 360 were applied to the output features of RBM, DBM and RNN models individually 361 only. A summary of the performance of RBM-based, DBM-based and RNN- 362 based anomaly detection schemes were presented in Tables 7. Furthermore, AUC 363 values between RNN-RBM, RNN, RBM and DBM-based anomaly detection ap- 364 proaches, and Standalone clustering algorithms were displayed in Figure 8 to fa- 365 cilitate interpretation of the obtained results. 366

The results from Table 7 demonstrated that the RNN-RBM-based OCSVM 367 monitoring algorithms clearly outperformed RBM, DBM and RNN-based tech- 368 niques. Indeed, RBM and DBM models omitted the presence of time dependence ${ }_{369}$ 
Table 7: Anomaly detection performances.

\begin{tabular}{llllrrrr}
\hline Scenario & Classifier & TPR & FPR & Accuracy & Precision & F1Score & AUC \\
\hline DBM & AGC & 0.90 & 0.98 & 0.31 & 0.32 & 0.47 & 0.46 \\
DBM & EM & 0.99 & 0.87 & 0.69 & 0.68 & 0.81 & 0.56 \\
DBM & KM & 0.99 & 0.87 & 0.68 & 0.66 & 0.80 & 0.56 \\
DBM & MS & 0.99 & 0.39 & 0.96 & 0.97 & 0.98 & 0.79 \\
DBM & OCSVM & 0.99 & 0.25 & 0.97 & 0.98 & 0.98 & 0.86 \\
DBM & SC & 0.97 & 0.94 & 0.45 & 0.44 & 0.60 & 0.51 \\
\hline RBM & AGC & 0.98 & 0.92 & 0.56 & 0.55 & 0.71 & 0.53 \\
RBM & EM & 0.96 & 0.71 & 0.94 & 0.97 & 0.97 & 0.62 \\
RBM & KM & 0.99 & 0.91 & 0.54 & 0.52 & 0.68 & 0.54 \\
RBM & MS & 0.99 & 0.90 & 0.57 & 0.55 & 0.71 & 0.54 \\
RBM & OCSVM & 0.99 & 0.22 & 0.98 & 0.98 & 0.99 & 0.88 \\
RBM & SC & 0.96 & 0.94 & 0.51 & 0.50 & 0.66 & 0.51 \\
\hline RNN & AGC & 0.94 & 0.96 & 0.53 & 0.55 & 0.69 & 0.49 \\
RNN & EM & 0.99 & 0.71 & 0.90 & 0.90 & 0.94 & 0.64 \\
RNN & KM & 0.99 & 0.77 & 0.85 & 0.85 & 0.92 & 0.60 \\
RNN & MS & 0.99 & 0.68 & 0.65 & 0.91 & 0.95 & 0.65 \\
RNN & OCSVM & 1.00 & 0.47 & 0.95 & 0.95 & 0.97 & 0.76 \\
RNN & SC & 0.98 & 0.92 & 0.53 & 0.52 & 0.68 & 0.52 \\
\hline RNN-RBM & AGC & 0.97 & 0.93 & 0.51 & 0.50 & 0.66 & 0.52 \\
RNN-RBM & EM & 0.97 & 0.51 & 0.95 & 0.97 & 0.97 & 0.72 \\
RNN-RBM & KM & 0.99 & 0.85 & 0.74 & 0.73 & 0.84 & 0.57 \\
RNN-RBM & MS & 0.99 & 0.29 & 0.97 & 0.98 & 0.98 & 0.84 \\
RNN-RBM & OCSVM & 0.99 & 0.03 & 0.99 & 0.99 & 0.99 & 0.98 \\
RNN-RBM & SC & 0.96 & 0.94 & 0.49 & 0.49 & 0.65 & 0.51 \\
\hline Standalone & AGC & 0.93 & 0.97 & 0.41 & 0.42 & 0.57 & 0.47 \\
Standalone & EM & 0.98 & 0.94 & 0.29 & 0.27 & 0.42 & 0.52 \\
Standalone & KM & 0.83 & 0.99 & 0.22 & 0.23 & 0.36 & 0.41 \\
Standalone & MS & 0.99 & 0.73 & 0.87 & 0.87 & 0.93 & 0.63 \\
Standalone & OCSVM & 1.00 & 0.38 & 0.97 & 0.97 & 0.98 & 0.80 \\
Standalone & SC & 0.99 & 0.91 & 0.53 & 0.51 & 0.67 & 0.54 \\
\hline & & & & & & &
\end{tabular}

in modeling time-series data. In other words, they were not designed to model 370 temporal dependency in data. Accordingly, important features and pattern in the 371 data were lost when these feature extractors (RBM and DBM). Also, it can be 372 highlighted that the RNN-RBM-based approach exhibited superior detection per- 373 formance compared to its counterpart RNN-based approach (Table 7). Amalga- 374 mating the desirable proprieties of RNN and RBM was helpful since this would 375 take account for the time dependencies in the multivariate data and thus lead to 376 a more representative model. Here, results also validated that the RNN-RBM- 377 


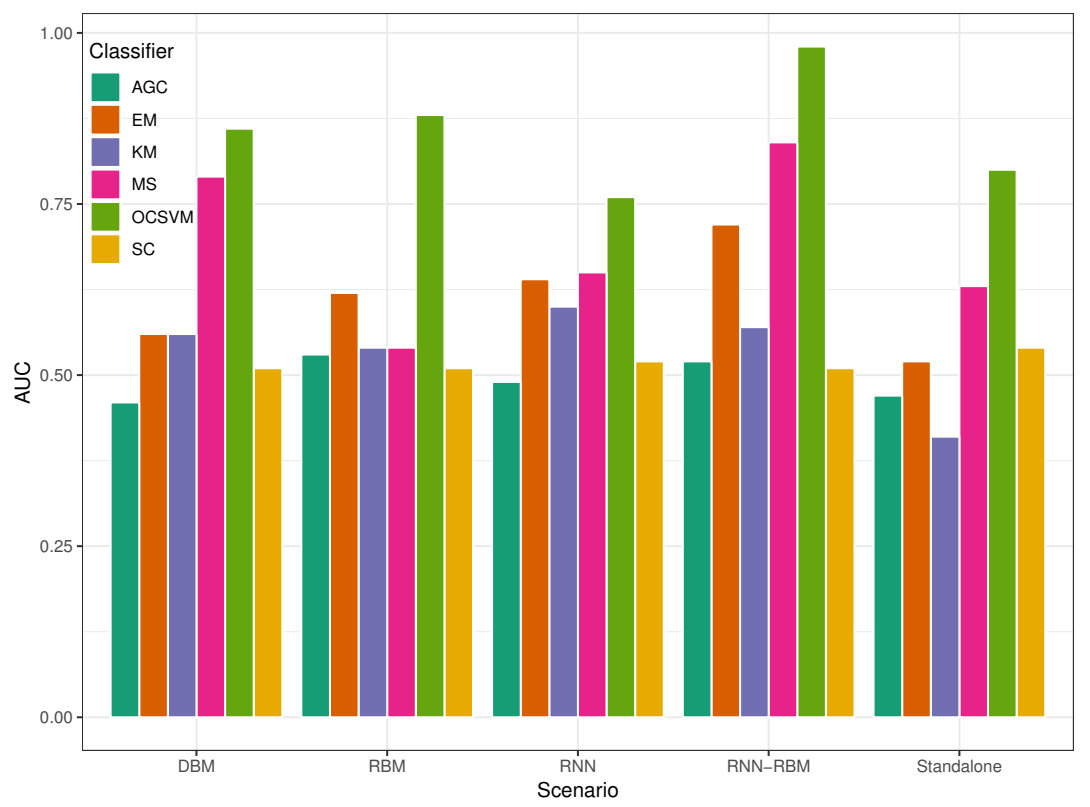

Figure 8: Area under the curve (AUC) values of proposed algorithms.

based approach, which was describing temporal dependencies, could also support 378 monitoring ICs of WWTPs. We believed this is related to the functionality that 379 RNN-RBM models approximated a complicated distribution for each time step. $\quad 380$

Furthermore, as expected, the detection results from the standalone clustering 381 techniques showed unsuitable performance for monitoring multivariate ICs data 382 (Table 7 and Figure 8). The performed experiments confirmed the superiority of ${ }_{383}$ recurrent neural networks combined with robust deep probabilistic features extrac- ${ }_{384}$ tor, RBM, against standalone machine learning techniques. Moreover, the results 385 from RNN-RBM-based OCSVM showed that the RNN-RBM model is proficient 386 in improving the detection of baseline approaches.

To monitor and detect abnormal influent conditions for sustainable and re- 388 silient wastewater treatment plant operations, we developed data-driven unsuper- 389 vised anomaly detection approaches based on deep learning methods and cluster- 390 ing algorithms. From the results presented, the following opinions were given. 391 First, methods integrating both RNN-RBM and clustering were more efficient 392 and accurate than the standalone clustering techniques. Second, the proposed al- 393 gorithms outperformed RBM and DBM-based clustering ones, which justified the 394 superior feature extraction quality of RNN-RBM models. It was presented that 395 
statistical one-class classifier trained in an unsupervised way (namely OCSVM) achieved higher accuracy compared to other adapted binary detectors (clustering algorithms). The robustness of OCSVM resided in its nonlinear kernel (e.g., 398 radial basis function or Gaussian function) was able to define hyperplane that sep- 399 arated normal observation from anomalies via the projection in different dimen- 400 sions or features space. Another crucial characteristic of OCSVM was its ability to 401 deal with high dimensionality which was the main bottleneck of many classifiers 402 (Schölkopf et al., 2001). The OCSVM proved its domination by outperforming ${ }_{403}$ other methods in all scenarios.

\section{Conclusion}

405

Data-driven unsupervised anomaly detection approaches based on deep learn- ${ }_{406}$ ing methods and clustering algorithms were developed in this study, in order to 407 monitor and detect influent conditions for sustainable and resilient wastewater ${ }_{408}$ treatment plant operations. The ability to discover and delineate temporal depen- 409 dencies in multivariate time series from RNN-RBM, and the sensitivity to identify 410 abnormal features from OCSVM were introduced and merged in this proposed 411 strategy. The efficiency of the proposed method were evaluated by real ICs data 412 from a coastal municipal WWTP in KSA. The approach involving recurrent neu- ${ }_{413}$ ral network-restricted Boltzmann machine coupled with one-class support vector ${ }_{414}$ machine produced the best efficiency and accuracy, compared to RBM or DBM- 415 based, or standalone clustering techniques. The RNN-RBM OCSVM detector ${ }_{416}$ was flexible for not requiring verification of assumptions on data distribution, and ${ }_{417}$ could be transferred straightforwardly and shared conveniently among various do- 418 main experts.

\section{References}

Álvarez-Cabria, M., Barquín, J., Peñas, F. J., 2016. Modelling the spatial and ${ }_{421}$ seasonal variability of water quality for entire river networks: Relationships 422 with natural and anthropogenic factors. Science of The Total Environment 545, 423 $152-162$.

Arthur, D., Vassilvitskii, S., 2007. k-means++: The advantages of careful seeding. 425 In: Proceedings of the eighteenth annual ACM-SIAM symposium on Discrete ${ }_{426}$ algorithms. Society for Industrial and Applied Mathematics, pp. 1027-1035. $\quad{ }_{427}$ 
Baruch, I. S., Georgieva, P., Barrera-Cortes, J., de Azevedo, S. F., 2005. Adaptive ${ }_{428}$ recurrent neural network control of biological wastewater treatment. Interna- ${ }_{429}$ tional Journal of Intelligent Systems 20 (2), 173-193.

430

Boulanger-Lewandowski, N., Bengio, Y., Vincent, P., 2012. Modeling temporal ${ }_{431}$ dependencies in high-dimensional sequences: Application to polyphonic music ${ }_{432}$ generation and transcription. arXiv preprint arXiv:1206.6392.

Boyd, G., Na, D., Li, Z., Snowling, S., Zhang, Q., Zhou, P., 2019. Influent fore- ${ }^{4} 34$ casting for wastewater treatment plants in north america. Sustainability 11 (6), 435 1764.

Capodaglio, A. G., Jones, H. V., Novotny, V., Feng, X., 1991. Sludge bulking ${ }_{437}$ analysis and forecasting: application of system identification and artificial neu- 438 ral computing technologies. Water Research 25 (10), 1217-1224. 439

Cheng, T., Harrou, F., Sun, Y., Leiknes, T., 2019. Monitoring influent mea- 440 surements at water resource recovery facility using data-driven soft sensor ap- ${ }^{441}$ proach. IEEE Sensors Journal 19 (1), 342-352.

Corominas, L., Garrido-Baserba, M., Villez, K., Olsson, G., Cortés, U., Poch, ${ }_{443}$ M., 2017. Transforming data into knowledge for improved wastewater treat- ${ }^{444}$ ment operation: A critical review of techniques. Environmental Modelling \& 445 Software.

Czuszynski, K., Ruminski, J., Kwasniewska, A., 2018. Gesture recognition with 447 the linear optical sensor and recurrent neural networks. IEEE Sensors Journal 448 18 (13), 5429-5438.

449

da Silva, W. F., dos Santos, I. F. S., de Oliveira Botan, M. C. C., Silva, A. P. M., 450 Barros, R. M., 2018. Reverse osmosis desalination plants in brazil: A cost anal- ${ }_{451}$ ysis using three different energy sources. Sustainable cities and society 43, 134- ${ }_{452}$ 143.

Dempster, A. P., Laird, N. M., Rubin, D. B., 1977. Maximum likelihood from ${ }_{454}$ incomplete data via the EM algorithm. Journal of the royal statistical society. ${ }_{455}$ Series B (methodological), 1-38.

Deutsch, J., He, D., 2018. Using deep learning-based approach to predict remain- ${ }_{457}$ ing useful life of rotating components. IEEE Transactions on Systems, Man, ${ }_{458}$ and Cybernetics: Systems 48 (1), 11-20. 
Dias, A., Alves, M., Ferreira, E., 2007. Application of computational intelligence 460 techniques for monitoring and prediction of biological wastewater treatment ${ }_{461}$ systems. In: IWA Conf. on Automation in Water Quality monitoring. pp. 1-8. ${ }_{462}^{462}$

Gibert, K., Horsburgh, J. S., Athanasiadis, I. N., Holmes, G., 2018a. Environmen- 463 tal data science. Environmental Modelling \& Software 106, 4-12. 464

Gibert, K., Izquierdo, J., Sànchez-Marrè, M., Hamilton, S. H., Rodríguez-Roda, ${ }_{465}$ I., Holmes, G., 2018b. Which method to use? an assessment of data mining 466 methods in environmental data science. Environmental Modelling \& Software ${ }_{467}$ 110, 3-27.

Haimi, H., Mulas, M., Corona, F., Vahala, R., 2013. Data-derived soft-sensors for ${ }_{469}$ biological wastewater treatment plants: An overview. Environmental modelling 470 \& software 47, 88-107.

Hill, D. J., Minsker, B. S., 2010. Anomaly detection in streaming environmental 472 sensor data: A data-driven modeling approach. Environmental Modelling \& 473 Software 25 (9), 1014-1022.

Honaker, J., King, G., 2010. What to do about missing values in time-series cross- 475 section data. American Journal of Political Science 54 (2), 561-581. 476

Honaker, J., King, G., Blackwell, M., et al., 2011. Amelia ii: A program for ${ }_{477}$ missing data. Journal of statistical software $45(7), 1-47$.

Kazor, K., Holloway, R. W., Cath, T. Y., Hering, A. S., 2016. Comparison of linear 479 and nonlinear dimension reduction techniques for automated process monitor- ${ }^{480}$ ing of a decentralized wastewater treatment facility. Stochastic environmental 481 research and risk assessment $30(5), 1527-1544$.

Khan, M., Nizami, A., Rehan, M., Ouda, O., Sultana, S., Ismail, I., Shahzad, K., ${ }_{483}$ 2017. Microbial electrolysis cells for hydrogen production and urban wastewa- 484 ter treatment: A case study of saudi arabia. Applied energy 185, 410-420. 485

Kim, M., Kim, Y., Kim, H., Piao, W., Kim, C., 2016. Evaluation of the k-nearest 486 neighbor method for forecasting the influent characteristics of wastewater treat- ${ }_{487}$ ment plant. Frontiers of Environmental Science \& Engineering 10 (2), 299-310. $\quad 488$

Lefers, R., Bettahalli, N. M. S., Nunes, S. P., Fedoroff, N., Davies, P. A., Leiknes, 489 T., 2016. Liquid desiccant dehumidification and regeneration process to meet 490 
cooling and freshwater needs of desert greenhouses. Desalination and Water 491 Treatment 57 (48-49), 23430-23442.

Li, J., Sharma, K., Liu, Y., Jiang, G., Yuan, Z., 2019. Real-time prediction of rain- ${ }^{493}$ impacted sewage flow for on-line control of chemical dosing in sewers. Water 494 research 149, 311-321.

Li, T., Winnel, M., Lin, H., Panther, J., Liu, C., O’Halloran, R., Wang, K., An, T., ${ }_{496}$ Wong, P. K., Zhang, S., et al., 2017. A reliable sewage quality abnormal event ${ }_{497}$ monitoring system. Water research 121, 248-257.

498

Lin, C.-H., Yu, R.-F., Cheng, W.-P., Liu, C.-R., 2012. Monitoring and control of uv 499 and uv-tio2 disinfections for municipal wastewater reclamation using artificial 500 neural networks. Journal of hazardous materials 209, 348-354.

Lorimer, P. A., Diec, V. M.-F., Kantarci, B., 2018. Covers-up: Collaborative verifi- ${ }_{502}$ cation of smart user profiles for social sustainability of smart cities. Sustainable 503 cities and society $38,348-358$.

Ma, X., Yu, H., Wang, Y., Wang, Y., 2015. Large-scale transportation network 505 congestion evolution prediction using deep learning theory. PloS one 10 (3), 506 e0119044.

Man, Y., Hu, Y., Ren, J., 2019. Forecasting cod load in municipal sewage based on 508 arma and var algorithms. Resources, Conservation and Recycling 144, 56-64. 509

Martens, J., Sutskever, I., 2011. Learning recurrent neural networks with hessian- 510 free optimization. In: Proceedings of the 28th International Conference on Ma- 511 chine Learning (ICML-11). Citeseer, pp. 1033-1040.

Newhart, K. B., Holloway, R. W., Hering, A. S., Cath, T. Y., 2019. Data-driven 513 performance analyses of wastewater treatment plants: A review. Water research. 514

Oneto, L., Fumeo, E., Clerico, G., Canepa, R., Papa, F., Dambra, C., Mazzino, 515 N., Anguita, D., 2017. Dynamic delay predictions for large-scale railway net- 516 works: Deep and shallow extreme learning machines tuned via thresholdout. 517 IEEE Transactions on Systems, Man, and Cybernetics: Systems 47 (10), 2754- 518 2767.

Qiao, J., Huang, X., Han, H., 2012. Recurrent neural network-based control for 520 wastewater treatment process. In: International Symposium on Neural Net- ${ }_{521}$ works. Springer, pp. 496-506. 
Salakhutdinov, R., Hinton, G., 2009. Deep Boltzmann machines. In: Artificial 523 Intelligence and Statistics. pp. 448-455.

Schölkopf, B., Platt, J. C., Shawe-Taylor, J., Smola, A. J., Williamson, R. C., 525 2001. Estimating the support of a high-dimensional distribution. Neural com- 526 putation 13 (7), 1443-1471.

Sheikhan, M., Jadidi, Z., Farrokhi, A., 2012. Intrusion detection using reduced- 528 size RNN based on feature grouping. Neural Computing and Applications ${ }_{529}$ 21 (6), 1185-1190.

Sutskever, I., Hinton, G. E., Taylor, G. W., 2009. The recurrent temporal restricted 531 boltzmann machine. In: Advances in Neural Information Processing Systems. $\quad 532$ pp. 1601-1608.

533

Sweeney, M. W., Kabouris, J. C., 2014. Modeling, instrumentation, automation, 534 and optimization of water resource recovery facilities. Water Environment Re- ${ }_{535}$ search 86 (10), 1314-1331.

Torregrossa, D., Castellet-Viciano, L., Hernández-Sancho, F., 2019. A data anal- 537 ysis approach to evaluate the impact of the capacity utilization on the energy 538 consumption of wastewater treatment plants. Sustainable cities and society 45, 539 307-313.

Venkatasubramanian, V., Rengaswamy, R., Kavuri, S. N., Yin, K., 2003. A re- ${ }^{541}$ view of process fault detection and diagnosis: Part iii: Process history based 542 methods. Computers \& chemical engineering 27 (3), 327-346.

543

Vincent, P., Larochelle, H., Lajoie, I., Bengio, Y., Manzagol, P.-A., 2010. Stacked 544 denoising autoencoders: Learning useful representations in a deep network with 545 a local denoising criterion. Journal of Machine Learning Research 11 (Dec), ${ }_{546}$ 3371-3408.

Wang, M., Li, H.-X., Chen, X., Chen, Y., 2016. Deep learning-based model re- ${ }_{548}$ duction for distributed parameter systems. IEEE Transactions on Systems, Man, 549 and Cybernetics: Systems 46 (12), 1664-1674.

Wilcox, S., Hawkes, D., Hawkes, F., Guwy, A., 1995. A neural network, based on 551 bicarbonate monitoring, to control anaerobic digestion. Water Research 29 (6), 552 1465-1470. 
Williams, R. J., Zipser, D., 1989. A learning algorithm for continually running fully recurrent neural networks. Neural computation 1 (2), 270-280.

554

555

Xiao, H., Huang, D., Pan, Y., Liu, Y., Song, K., 2017. Fault diagnosis and progno- 556 sis of wastewater processes with incomplete data by the auto-associative neural ${ }_{557}$ networks and arma model. Chemometrics and Intelligent Laboratory Systems 558 $161,96-107$.

559

Xiao, L., Zhang, Z., Li, S., 2018. Solving time-varying system of nonlinear equa- 560 tions by finite-time recurrent neural networks with application to motion track- 561 ing of robot manipulators. IEEE Transactions on Systems, Man, and Cybernet- 562 ics: Systems.

563

Yang, Y., Wu, Q. J., Wang, Y., 2018. Autoencoder with invertible functions for ${ }_{564}$ dimension reduction and image reconstruction. IEEE Transactions on Systems, 565 Man, and Cybernetics: Systems 48 (7), 1065-1079.

566

Yoshihara, A., Fujikawa, K., Seki, K., Uehara, K., 2014. Predicting stock market ${ }_{567}$ trends by recurrent deep neural networks. In: Pacific rim international confer- 568 ence on artificial intelligence. Springer, pp. 759-769.

569

Yuan, Z., Olsson, G., Cardell-Oliver, R., van Schagen, K., Marchi, A., Deletic, 570 A., Urich, C., Rauch, W., Liu, Y., Jiang, G., 2019. Sweating the assets-the 571 role of instrumentation, control and automation in urban water systems. Water 572 research.

573

Zhu, J.-J., Anderson, P. R., 2017. Effect of long-term successive storm flows on 574 water reclamation plant resilience. Water research 111, 1-9. 575

Zhu, J.-J., Kang, L., Anderson, P. R., 2018. Predicting influent biochemical oxy- 576 gen demand: Balancing energy demand and risk management. Water research 577 128, 304-313.

578 